\title{
Time-varying on-body wireless channel model during walking
}

\author{
Michael Cheffena
}

\begin{abstract}
A novel dynamic channel model for on-body wireless communication during walking is proposed. The developed model utilizes a human walking model which provides detailed information on the movement of the human body parts. The diffraction of the signal around the body parts is used to describe the time-varying shadowing effects. Body part movements are also used to estimate the signal fading caused by angular variations of the transmitting and receiving antenna gains. A Rice distribution is used to represent the multipath fading effects caused by objects around the human body. Simulation results of the first- and second-order statistics of the received signal affected by moving body parts for $2.4 \mathrm{GHz}$ signal are presented. To illustrate the capabilities of the developed model, time series were generated and used in system performance calculations. The obtained results give an insight into the potential advantages of link diversity technique in wireless body area networks (WBANs).
\end{abstract}

Keywords: Body area network; On-body communication; Fading channels

\section{Introduction}

In recent years, body area networks are gaining increasing attention because of their potential applications in various domains such as health, entertainment, and sports. The use of wireless communication in the immediate vicinity of the human body eliminates the need for wired interconnections and hence the concept of wireless body area network (WBAN).

However, various propagation measurement results have shown that the on-body wireless channel is subject to fading caused by the movement of the human body [1-9]. In addition to the shadowing of the signal by moving body components, signal reflection/scattering from objects around the human body result in multipath fading effects $[1,2,10]$. Furthermore, the angular variations of the antenna gains during walking give rise to time-varying channel conditions $[9,11,12]$. Understanding the on-body propagation channel is thus important for successful design of WBAN systems.

A number of studies on the signal fading caused by the movement of the human body are reported in the literature, most of them are based on radio frequency (RF) measurements [1-9] or using numerical simulations

Correspondence: michael.cheffena@hig.no

Gjøvik University College, Teknologivn. 22, Gjøvik N-2815, Norway such as finite-difference time-domain (FDTD) [10,13-15]. A finite-state Markov model for dynamic on-body channels, where the model parameters are extracted from the RF measurements is reported in [5]. A two-state alternating Weibull renewal process for describing the dynamical properties of the on-body channel is proposed in [6]. In addition, by modeling the trunk, arms, and legs of a human body as infinite cylinders, Liu et al. [7] proposed a method for calculating the scattering signal by body components. A series of time-consecutive scenarios with different positions of the arms at the azimuth is included to describe the time-varying behavior of the channel. In [15], a phantom created by an animation software is used for simulating the time-varying on-body communication channel. Similar study is conducted in [16] to characterize the shadowing properties of an arm-waving human body.

In this paper, a novel dynamic channel model for onbody wireless communication during walking is proposed by utilizing a human walking model. Using geometrical relations, the diffraction of the signal around body parts is calculated to describe the time-varying shadowing effects. The movements of the body parts are also used to estimate the signal fading caused by angular variations of the antenna gains. In addition, a Rice distribution is used to represent the multipath fading effects caused by objects around the human body. To show the potential of the

\section{Springer}

(C) 2014 Cheffena; licensee Springer. This is an Open Access article distributed under the terms of the Creative Commons Attribution License (http://creativecommons.org/licenses/by/2.0), which permits unrestricted use, distribution, and reproduction in any medium, provided the original work is properly cited. 
proposed model, time series were generated and used in system performance calculations. The results give an insight into the advantages of link diversity technique in WBANs.

The paper begins by discussing the human walking model in Section 2. The proposed dynamic channel model for on-body wireless communication is presented in Section 3. Numerical results and discussions are given in Section 4. Finally, the conclusions are given in Section 5.

\section{The human model}

Human walking models have been developed for use in, e.g., virtual reality [17-19]. Such models may provide detailed informations on the movement of human body parts which are necessary in characterizing the time-varying on-body wireless channel. Figure 1 shows a human body model consisting of 12 body parts which are modeled as cylinders except the head which is modeled as a sphere. The body parts are connected to each other by translations and rotations (see Table 1 for description) and flex as the person walks. These time-dependent body part translations and rotations have to be known in order to characterize the time-varying on-body wireless channel.

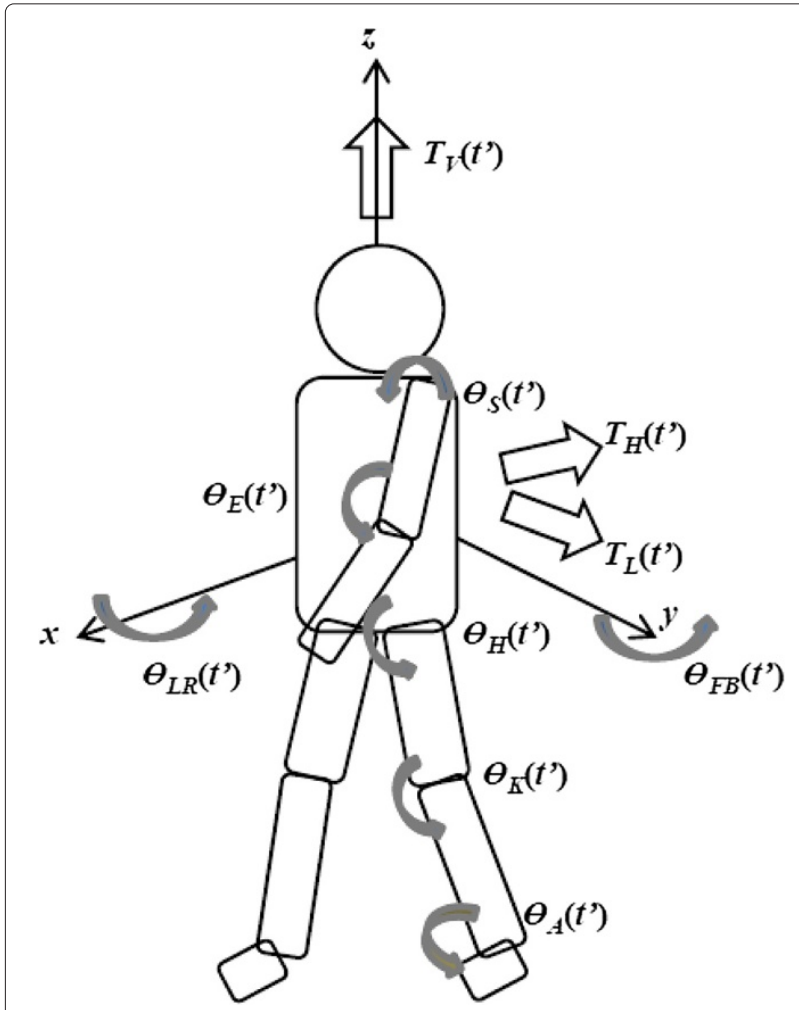

Figure 1 Human body model with translations and rotations (see Table 1) [19,20].
Table 1 Body part translations and rotations (see Figure 1)

\begin{tabular}{lc}
\hline Lateral translation & $T_{\mathrm{L}}\left(t^{\prime}\right)$ \\
Horizontal translation & $T_{\mathrm{H}}\left(t^{\prime}\right)$ \\
Vertical translation & $T_{\mathrm{V}}\left(t^{\prime}\right)$ \\
Forward/backward angle & $\theta_{\mathrm{FB}}\left(t^{\prime}\right)$ \\
Left/right angle & $\theta_{\mathrm{LR}}\left(t^{\prime}\right)$ \\
Shoulder angle & $\theta_{\mathrm{S}}\left(t^{\prime}\right)$ \\
Elbow angle & $\theta_{\mathrm{E}}\left(t^{\prime}\right)$ \\
Hip angle & $\theta_{\mathrm{H}}\left(t^{\prime}\right)$ \\
Knee angle & $\theta_{\mathrm{K}}\left(t^{\prime}\right)$ \\
Ankle angle & $\theta_{\mathrm{A}}\left(t^{\prime}\right)$ \\
\hline
\end{tabular}

Based on experimental data, Boulic et al. [19] developed a model for calculating the time-dependent body part translations and rotations. In the model, a walking cycle begins with left foot heel strike (HS), continues though right foot HS, left foot toe off (TO), and ends with the second HS of the left foot (see Figure 2). The velocity of human walking in meters per second is given by

$$
v=v_{\mathrm{r}} h_{\mathrm{t}},
$$

where $v_{\mathrm{r}}$ is the relative velocity in thigh height per second, and $h_{\mathrm{t}}$ is the thigh height. The relative velocity depends on the cycle length $l(\mathrm{~m})$ and cycle frequency $f(\mathrm{~Hz})$ of the human walking as

$$
v_{\mathrm{r}}=l f,
$$

where a cycle frequency $f$ is the number of cycles per second, and one cycle is defined as two contacts of the left heel with the floor. The relationship between the relative velocity and the cycle length of a normal walking person is expressed as [19]

$$
l=1.346 \sqrt{v_{\mathrm{r}}} \text {. }
$$

The different angle trajectories and translations shown in Figure 1 can be expressed as function of the relative time, $t^{\prime}$, which is defined as the time between zero and one human cycle, $t^{\prime}=|t f|_{\bmod 1}$. They are synchronized with the left human body motion, and the origin is at the left heel strike. Displacements of the right human body parts are obtained by a phase displacement of half a cycle, i.e., $t^{\prime}=|t f+\phi|_{\text {mod } 1}$.

The position of all body parts is calculated relative to the spine center (i.e., the center of the coordinate system shown in Figure 1). The vertical translation $T_{\mathrm{V}}\left(t^{\prime}\right)$, lateral translation $T_{\mathrm{L}}\left(t^{\prime}\right)$, and horizontal $T_{\mathrm{H}}\left(t^{\prime}\right)$ translation situate the body relatively to the average position it has while moving with the velocity $v_{\mathrm{r}}$. These are empirical relations found in [19]. The lateral translation is positive on the left-hand side of the human body and is given by the expression. 


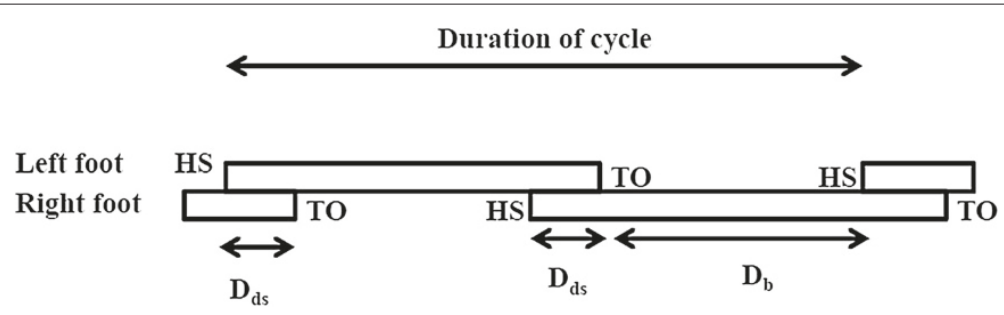

Figure 2 Temporal structure of the walking cycle. $\mathrm{HS}=$ heel strike, $\mathrm{TO}=$ toe off, $D_{\mathrm{ds}}=$ duration of double support, and $D_{\mathrm{b}}=$ duration of balance.

For $v_{\mathrm{r}}>0.5$,

$$
T_{\mathrm{L}}\left(t^{\prime}\right)=-0.032 \sin \left[2 \pi\left(t^{\prime}-0.1\right)\right]
$$

For $v_{\mathrm{r}} \leq 0.5$,

$$
T_{\mathrm{L}}\left(t^{\prime}\right)=\left(-0.128 v_{\mathrm{r}}^{2}+0.128 v_{\mathrm{r}}\right) \sin \left[2 \pi\left(t^{\prime}-0.1\right)\right] .
$$

The horizontal and vertical translations have equivalent expressions. The pelvis rotates in forward/backward, left/right, and torsion rotation as the person walks. The forward/backward rotation of the pelvis moves the center of gravity of the body in order to help the forward motion of the leg and is expressed as,

$$
\text { for } v_{\mathrm{r}}>0.5 \text {, }
$$

$$
\theta_{\mathrm{FB}}\left(t^{\prime}\right)=-2+2 \sin \left[2 \pi\left(2 t^{\prime}-0.1\right)\right] .
$$

For $v_{\mathrm{r}} \leq 0.5$,

$\theta_{\mathrm{FB}}\left(t^{\prime}\right)=8 v_{\mathrm{r}}^{2}-8 \mathrm{v}_{r}+\left(-8 v_{\mathrm{r}}^{2}+8 v_{\mathrm{r}}\right) \sin \left[2 \pi\left(2 t^{\prime}-0.1\right)\right]$.

Equivalent empirical expressions for the left/right and torsion rotations are also given in [19]. The trajectories of flexing for the elbow, hip, knee, ankle, and thorax are modeled using cubic spline passing through control points (for a given relative velocity) located at the extremities of the trajectories. These control points define the relative time and angle of the rotations and are functions of the relative velocity on the following three ranges:

$$
\begin{aligned}
\text { i } & 0 \leq v_{\mathrm{r}}<0.5 \text { - from the rest position to reach a slow } \\
& \text { gait } \\
\text { ii } & 0.5 \leq v_{\mathrm{r}}<1.3 \text { - walking at almost constant speed } \\
\text { iii } & 1.3 \leq v_{\mathrm{r}}<3 \text { - walking fast. }
\end{aligned}
$$

For example, the position of the human left lower arm is a translation to the left shoulder, a rotation of the left shoulder joint, a translation to the left elbow, a rotation of the left elbow, and a translation to the center of the lower arm [20]. The rotation of the left shoulder is given by [19]

$$
\theta_{\mathrm{S}}\left(t^{\prime}\right)=-3-9.88 v_{\mathrm{r}}\left(0.5+\cos \left[2 \pi t^{\prime}\right]\right) .
$$

A positive value indicates a front swinging motion. The left shoulder has a $-3^{\circ}$ rest position. The rotation of the left elbow is composed of three control points. The control points define the elbow rotation, $\theta_{\mathrm{E}}\left(t^{\prime}\right)$. Using Hermit spline functions, the trajectory is constructed by connecting the three control points. The relative time coordinates and rotations of the three control points are shown in Figure $3 \mathrm{a}, \mathrm{b}$. The rotation of the elbow, $\theta_{\mathrm{E}}\left(t^{\prime}\right)$, obtained using cubic spline passing through the control points is shown in Figure 3c. Equivalent control points for the hip, knee, ankle, and thorax are given in [19], which are used to define their angular rotations. See [19] for details on the human walking model. Figure 4 shows the displacements and angular variations of the different body parts (left side) for one walking cycle with relative velocity, $v_{\mathrm{r}}=1$, calculated using the walking model. Variations of the right human body parts are obtained by a phase displacement of half a cycle. In the following, these time-dependent body part translations and rotations are used to characterize the time-varying on-body wireless channel during walking.

\section{The propagation model}

Various propagation measurement results have shown that the on-body wireless channel is subject to fading caused by the movement of the human body [1-9]. One of the main propagation impairment is the shadowing of the signal by the human body. Figure 5 shows the position of the left arm at different time instances during walking. Assuming the transmitting antenna ( $\mathrm{Tx}$ ) is placed on the belt toward the right side of the body and the receiving antenna (Rx) mounted on the left wrist, we can observe that the path between $\mathrm{Tx}$ and $\mathrm{Rx}$ antennas is shadowed (i.e., non-line-of-sight (LOS) path) by the upper body at position B, but not shadowed (i.e., line-of-sight (LOS) path) at positions $\mathrm{A}$ and $\mathrm{C}$. Thus, as the person continue walking, the signal will be subject to periodic shadowing effects caused by the shadowing of the LOS path by the human body. In addition, the received reflected signals 


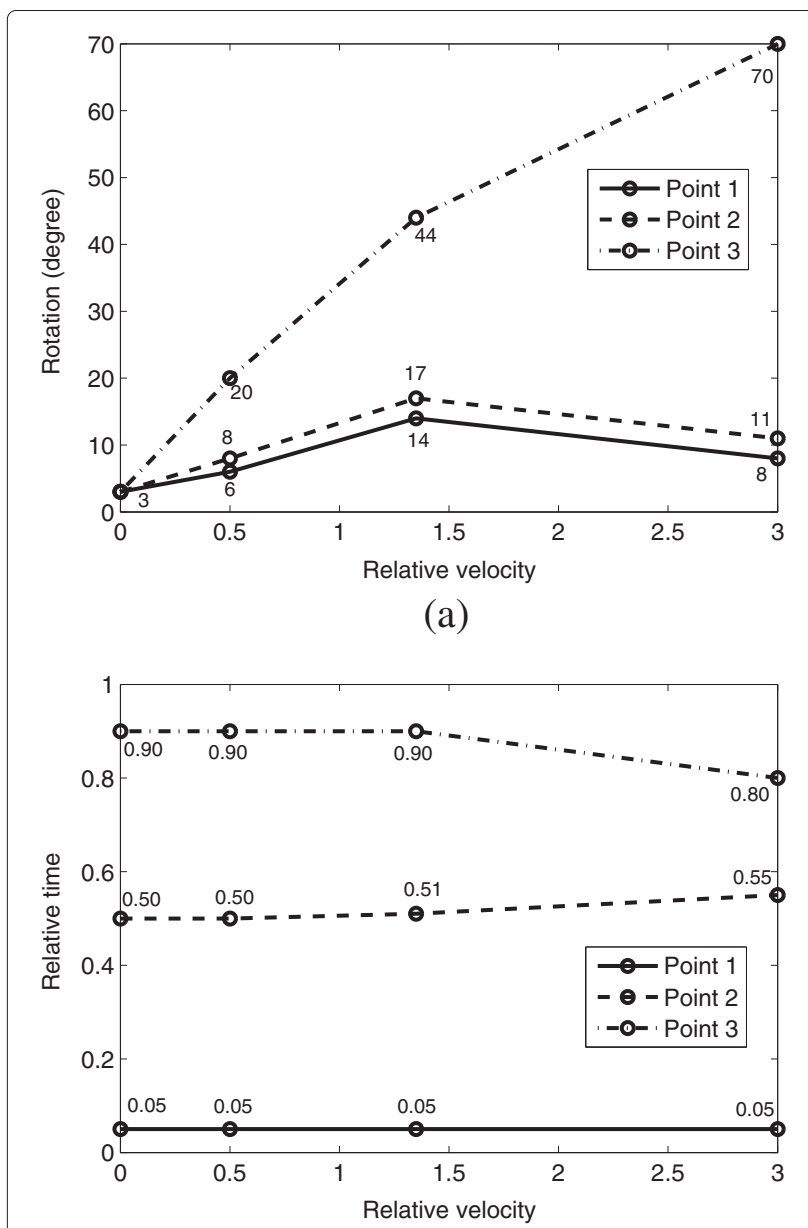

(b)

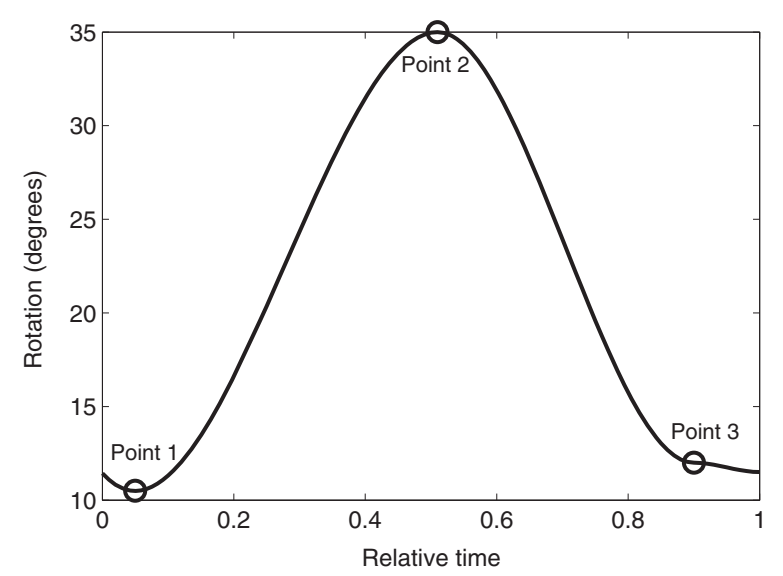

(c)

Figure 3 Elbow angular rotation with relative velocity of $v_{r}=1$ obtained using cubic spline passing though control points. (a) Control point rotation function. (b) Control point relative time function. (c) Elbow angular rotation. from different objects (floor, ceiling, walls, furnitures, etc.) around the human body cause multipath fading effects. The movement of body parts create time-varying channel conditions. Characterization of the Doppler spectra is thus important for the determination of the time variance of the on-body wireless channel. These different propagation impairments have to be combined to yield a complete channel model for on-body wireless communication. In subsequent sections, we discuss each of the aforementioned propagation mechanisms for signal effect caused by the movement of the human body during walking and combine them to give a complete channel model.

\subsection{Shadowing}

As discussed above, the movements of the body parts cause time-varying shadowing effects on the received signal. This is mainly due to the obstruction of the LOS path by the body parts. Only diffracted fields are present in the shadow region. Figure 6 shows an example where the transmitting antenna (Tx) is placed on the belt toward the right side of the body and the receiving antenna $(\mathrm{Rx})$ mounted on the left wrist. During walking, the positions of the Tx and Rx antennas vary in accordance to the angular variations of the corresponding body parts shown in Figure 4 (for $\left.v_{\mathrm{r}}=1\right)$. The time-varying diffracted signal can then be calculated using Kirchhoff diffraction equation $[21,22]$ which gives the relationship between the aperture diffracted electric field at an observation point, $P$, and the free-space electric field

$$
\frac{E(P)}{E_{o}(P)}=\frac{j}{2} \iint_{\Sigma(u, v)} \exp \left(-j \frac{\pi}{2}\left(u^{2}+v^{2}\right)\right) d u d v
$$

with

$$
\left[\begin{array}{l}
u \\
v
\end{array}\right]=\frac{\sqrt{2}}{R_{1}}\left[\begin{array}{l}
x_{o} \\
z_{o}
\end{array}\right]
$$

where $O$ is the intersection point (with Cartesian coordinates $x_{o}$ and $z_{o}$ ) of the Tx-Rx straight line with the obstacle (see Figure 6). Parameters $x_{o}$ and $z_{o}$ are position dependent and vary during walking. Parameter $R_{1}$ is the radius of the first Fresnel zone given by

$$
R_{1}=\sqrt{\frac{\lambda a b}{a+b}},
$$

where $a$ and $b$ are the distances from the transmitter and the receiver to the aperture plane. For calculating the double integral in (9), the human body surface has to be split into three separate, contiguous surfaces $\left(\Sigma_{1}, \Sigma_{2}\right.$, and $\left.\Sigma_{3}\right)$ to facilitate the calculation [23]. These surfaces vary with time as the person walks. Table 2 shows the integral limits for parameters $u$ and $v$ of the three surfaces 


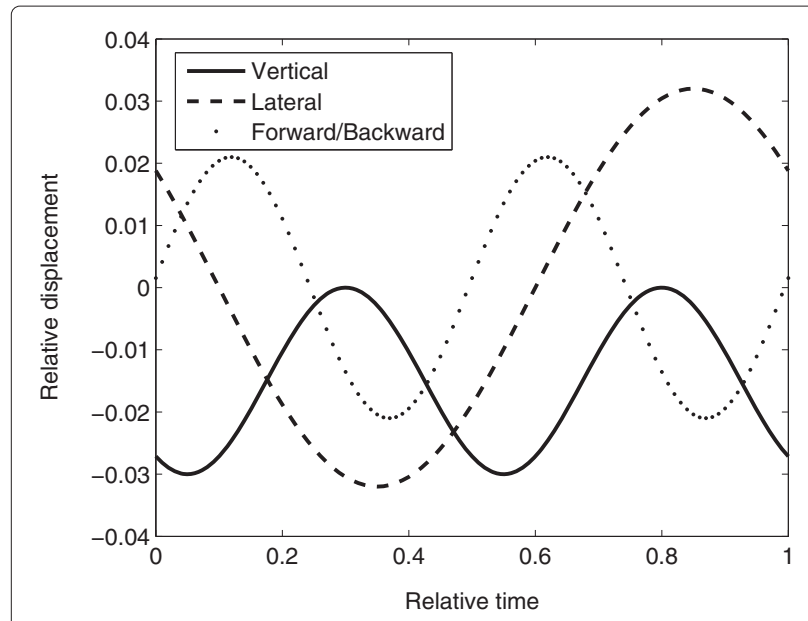

(a)

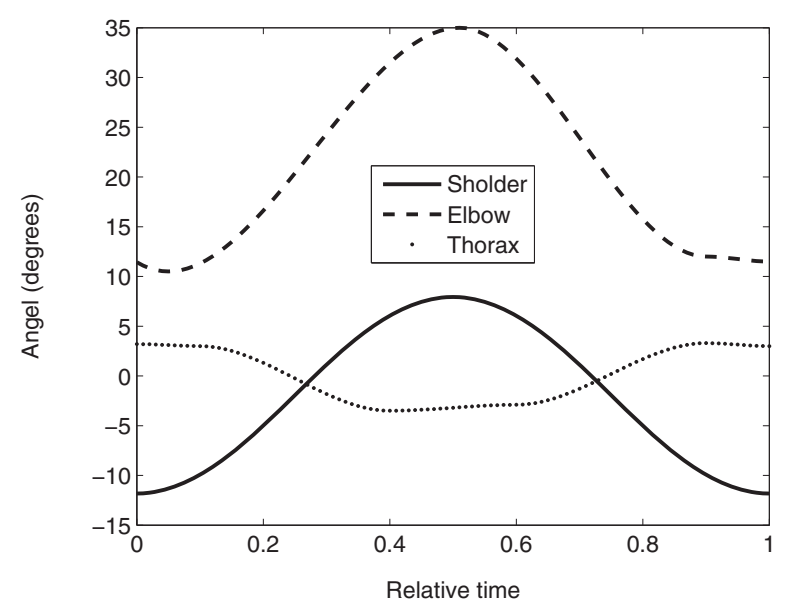

(c)

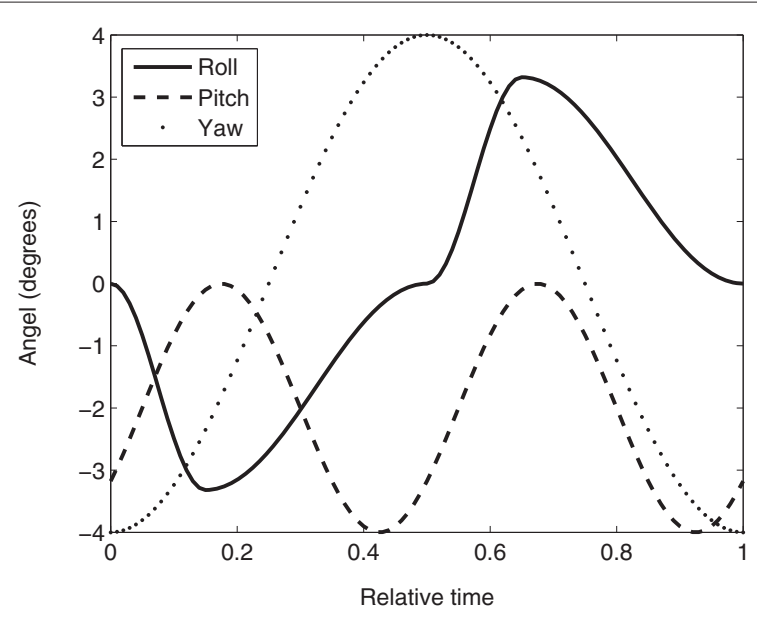

(b)

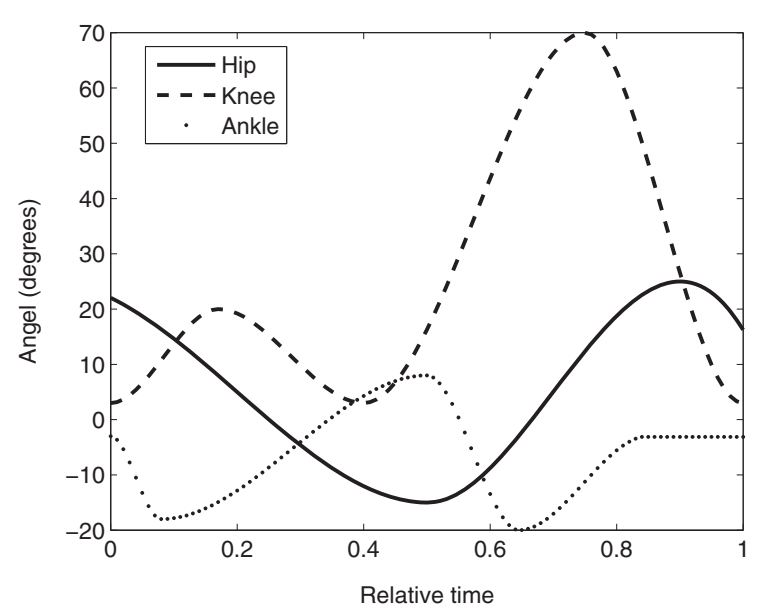

(d)

Figure 4 Variations of the different body parts (left side) for one walking cycle with relative velocity $\boldsymbol{v}_{\mathbf{r}}=\mathbf{1}$. Variations of the right human body parts are obtained by a phase displacement of half a cycle. (a) Body displacements. (b) Pelvic angular variations. (c) Angular variations of the left shoulder, elbow, and thorax. (d) Angular variations of the left hip, knee, and ankle.
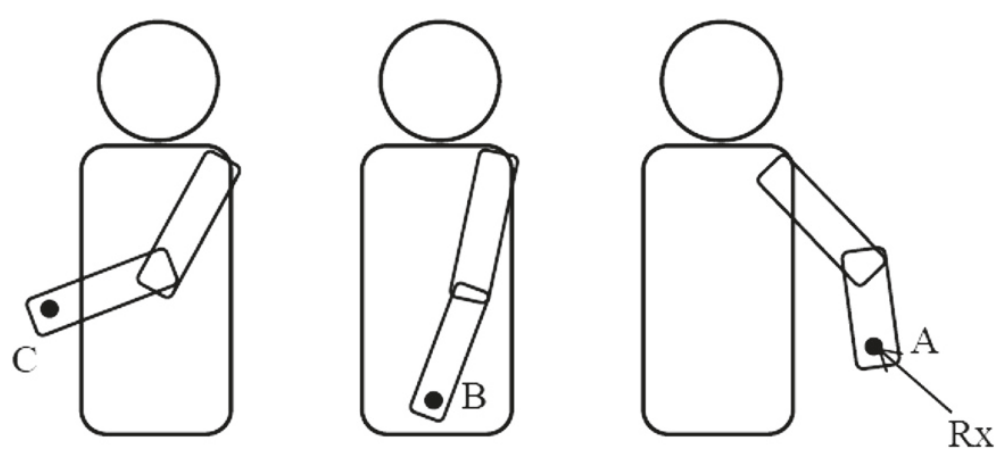

Figure 5 The position of the left arm at different time instances during walking. The transmitting antenna (Tx) is placed on the belt toward the right side of the body and the receiving antenna (Rx) mounted on the left wrist. 


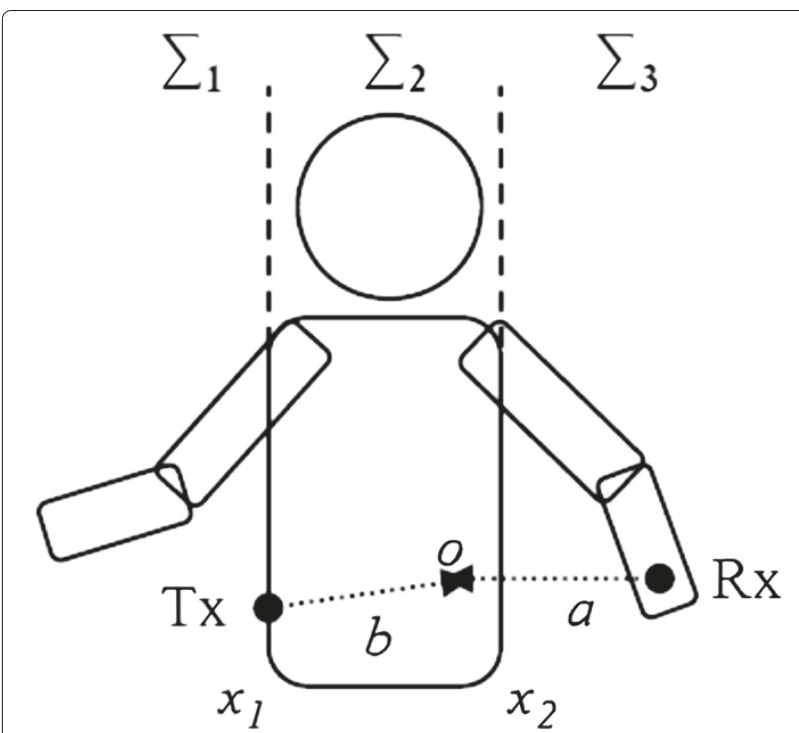

Figure 6 Example of signal body diffraction. The transmitting antenna $(T x)$ is placed on the belt toward the right side of the body and the receiving antenna $(\mathrm{Rx})$ mounted on the left wrist. Parameters $\Sigma_{1}, \Sigma_{2}$, and $\Sigma_{3}$ are surfaces of the aperture. Parameter $O$ is the intersection point of the $T x-R x$ straight line with the body part. Parameters $x_{1}$ and $x_{2}$ are the start and end of $\Sigma_{2}$ along the $x$-axis, respectively.

(where $x_{1}$ and $x_{2}$ are the start and end of $\Sigma_{2}$ along the $x$ axis, respectively, and $h$ is the height of the person), see Figure 6.

The total received diffracted field is then the combination of the three aperture diffracted fields whose surfaces are $\Sigma_{1}, \Sigma_{2}$, and $\Sigma_{3}$ with assumed infinite depth. The transitions between LOS and NLOS conditions are not sharp and thus more realistic regarding the empirical results. Using this model, good estimation of the shadow region behind a human body part might be obtained.

\subsection{Multipath fading}

The shadowing effect discussed above represent the large scale variation of the signal caused by the movements of the human body parts. However, a fast-fading component is also present which is mainly caused by the reflection of the signal from objects around the human body (floor, ceiling, walls, furnitures, etc.). Reported measurement

Table 2 Integral limits for parameter $u$ and $v$ of the three surfaces [23]

\begin{tabular}{cccc} 
& $\boldsymbol{\Sigma}_{\mathbf{1}}$ & $\boldsymbol{\Sigma}_{\mathbf{1}}$ & $\boldsymbol{\Sigma}_{\mathbf{3}}$ \\
\hline$u$ & $-\infty, \sqrt{2} \frac{x_{1}-x_{0}}{R_{1}}$ & $\sqrt{2} \frac{x_{1}-x_{0}}{R_{1}}, \sqrt{2} \frac{x_{2}-x_{0}}{R_{1}}$ & $\sqrt{2} \frac{x_{2}-x_{0}}{R_{1}}, \infty$ \\
$v$ & $-\sqrt{2} \frac{z_{0}}{R_{1}}, \infty$ & $\sqrt{2} \frac{h-z_{0}}{R_{1}}, \infty$ & $-\sqrt{2} \frac{z_{0}}{R_{1}}, \infty$ \\
\hline
\end{tabular}

results show that the multipath fading distribution follows a Rice distribution [1], expressed as

$$
p(r)=\frac{r}{\sigma^{2}} \exp \left\{\frac{-\left(r^{2}+s^{2}\right)}{2 \sigma^{2}}\right\} I_{0}\left(\frac{r s}{\sigma^{2}}\right),
$$

where $s$ is the magnitude of the coherent component, and $\sigma^{2}$ is the variance of the incoherent component.

The Rice K-factor $\left(K=s^{2} / 2 \sigma^{2}\right)$ depends on the mobility of the human body. High value of Rice K-factor might be expected when the human subject is at rest (i.e., a strong main signal path exists). As a result, there might be little fast fading which is mainly caused by, e.g., involuntary breathing movements. However, low K-factor is expected in walking scenario as the link between the transmitter and the receiver is shadowed by the body and propagation occurs by reflections from the surrounding environments [1].

\subsection{Doppler shift}

The movement of body parts create time-varying channel conditions. Characterization of the Doppler spectra is thus important for the determination of the time variance of the on-body wireless channel. The situation where the antenna is moving in a random environment leads to the classical Jakes spectrum (with bathtub-like shape) for scatters uniformly distributed in azimuth [24]. For the case where the antenna is stationary, moving scatterers in the channel will lead to a different Doppler spectrum which peaks at $0 \mathrm{~Hz}$ and falls off rapidly $[25,26]$.

For electrically large scatterer such as the human body part with scattering pattern peaking in the forward direction, the Doppler spectrum is given by [27]

$$
S_{n}(f)=(-1)^{n} P_{n-0.5}\left(\frac{f^{2}}{2}-1\right),
$$

where $P_{n-0.5}$ is the $n$th order Legendre function. Based on measurements, a simplified expression of the normalized Doppler spectrum for moving body parts was found in [7], expressed as

$$
S(f)=\frac{1}{\gamma+f^{2}},
$$

where the measured value of parameter $\gamma$ and $-20 \mathrm{~dB}$ Doppler bandwidth (BW) for radio links between different body parts is shown in Table 3. We can observe from Table 3 that slow movements are represented by small values of the parameter $\gamma$.

\subsection{The overall simulation model}

During walking, the overall signal fading is due to positiondependent diffracted fields (discussed in Section 3.1), 
Table 3 Doppler spectrum parameter for on-body wireless channel [7]

\begin{tabular}{lcc}
\hline Radio link & BW $\mathbf{( H z )}$ & $\boldsymbol{\gamma}$ \\
\hline Hip-chest & 1.5 & 0.004 \\
Hip-thigh & 6.4 & 0.067 \\
Hip-wrist & 7.3 & 0.094 \\
Hip-foot & 10 & 0.231 \\
Left ear-hip & 1.3 & 0.007 \\
Left ear-wrist & 8 & 0.574 \\
Left ear-foot & 12.6 & 0.862 \\
Left ear-right ear & 0.3 & 0.013 \\
\hline
\end{tabular}

multipath fading effects caused by reflection/scattering of the signal by objects around the human body (discussed in Section 3.2), and Doppler effects caused by the movement of body parts (discussed in Section 3.3). Figure 7 shows the overall simulation model for the on-body wireless channel during walking. In the model, a complex white Gaussian noise is passed to the filter defined in (14) to shape the Doppler spectrum. The filter outputs are multiplied by the standard deviation of the incoherent component, $\sigma$, of (12). Then, the magnitude of the coherent component, $s$, is added to obtain the Rice distributed multipath fading effects. Using (9) and (10), the position-dependent diffracted fields are calculated by utilizing the human walking model discussed in Section 2. The multipath fading effects and the diffracted fields are summed and then weighted by the time-varying antenna gains of the transmitter $G_{\mathrm{Tx}}$ and receiver $G_{\mathrm{Rx}}$ antennas to obtain the complex signal envelope for the on-body wireless channel during walking.

The influence of body movement on antenna orientation strongly depends on its location on the body, which is related to the movement of a specific body segment. Given the gain pattern of an antenna, $G(\theta, \phi)$, the gain variation can be calculated using the angular variation of the corresponding body part during waking [28].

$$
G_{t^{\prime}}(\theta, \phi)=G\left(\theta+\delta \theta_{t^{\prime}}, \phi+\delta \phi_{t^{\prime}}\right),
$$

where $\theta$ and $\phi$ are the elevation and azimuth angles of the antenna, respectively. Parameters $\delta \theta_{t^{\prime}}$ and $\delta \phi_{t^{\prime}}$ describe the change in the orientation of the antenna due to the movement of the body part where the antenna is mounted and are calculated from the walking model discussed in Section 2.

\section{Numerical results and discussions}

\subsection{Simulations}

In the simulations, the transmitting antenna is placed on the belt toward the right side of the body (representing where a mobile phone might be worn), and two receiver antenna positions are considered, one on the left wrist (e.g., wristwatch providing incoming call alert or a control device [15]) and the other on the left ankle (e.g., a training aid device). These receiver locations are of interest as the wrist and ankle movements are large and are mostly in NLOS position during walking (in general, the Tx and $R x$ antennas can be mounted anywhere in the body). A human body dimension shown in Table 4 is used in our analysis. The values in Table 4 are of the form $(x$, $y$, and $z$ ) in $\mathrm{cm}$ with reference to the coordinate system shown in Figure 1. The simulation parameters are given in Table 5. During walking, the variations of the transmitting and receiving antenna positions are found using the human walking model described in Section 2 (for the corresponding body parts where the Tx and Rx antennas are mounted). These antenna positions are further used in (9)

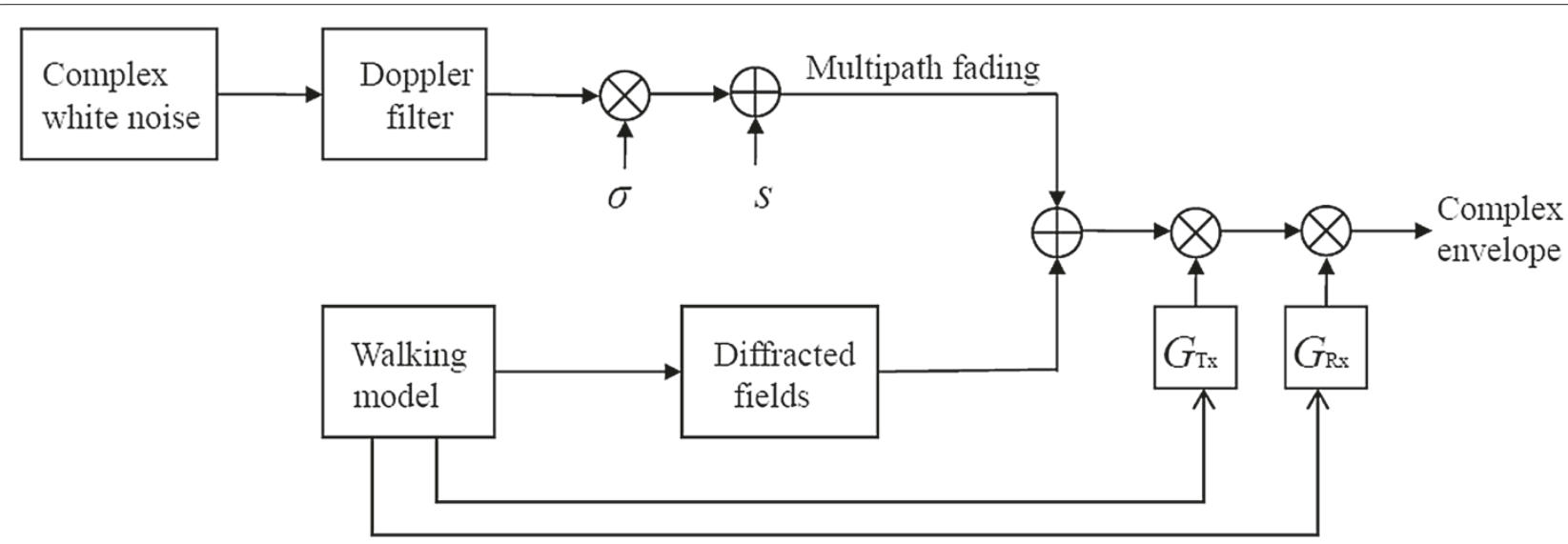

Figure 7 Simulation model for the time-varying on-body wireless channel during walking. Parameters $\sigma$ and $s$ are the standard deviation of the incoherent and the magnitude of the coherent component of the Rice distribution defined in (12), respectively. Parameters $G_{T x}$ and $G_{R x}$ are the transmitting and receiving antenna gains, respectively. 
Table 4 Human body dimensions in centimeters

\begin{tabular}{lccc}
\hline Body part & Right $(\boldsymbol{x}, \boldsymbol{y}, \boldsymbol{z})$ & Left $(\boldsymbol{x}, \boldsymbol{y}, \boldsymbol{z})$ & Body $(\boldsymbol{x}, \boldsymbol{y}, \boldsymbol{z})$ \\
\hline Shoulder & $(0,-21.6,29.4)$ & $(0,21.6,29.4)$ & - \\
Elbow & $(0,-21.6,-9.9)$ & $(0,21.6,-9.9)$ & - \\
Fingers & $(0,-21.6,-44.1)$ & $(0,-21.6,-44.1)$ & - \\
Hip & $(0,-17.8,-17.8)$ & $(0,17.8,-17.8)$ & - \\
Knee & $(0,-17.8,-64.4)$ & $(0,17.8,-64.4)$ & - \\
Ankle & $(0,-17.8,-117.8)$ & $(0,17.8,-117.8)$ & - \\
Toe & $(20.3,-17.8,-117.8)$ & $(20.3,17.8,-117.8)$ & - \\
Spine center & - & - & $(0,0,0)$ \\
Throat bottom & - & - & $(0,0,29.5)$ \\
Throat top & - & - & $(0,0,39.7)$ \\
Head center & - & - & $(0,0,53.1)$ \\
Head top & - & - & $(0,0,65.2)$ \\
\hline
\end{tabular}

and (10) to calculate the time-varying diffracted signals caused by the human body. Note that parameters $x_{o}$ and $z_{o}$ in (10) are position dependent and vary according to the walking model described in Section 2. Furthermore, a Rice-distributed signal with K-factor of $7 \mathrm{~dB}$ is used in the simulations to represent the fast fading (multipath) component of the received signal.

Simulated time series of the received signal at $2.4 \mathrm{GHz}$ for the right-belt-to-left-wrist and right-belt-to-left-ankle links for one walking cycle calculated using the method described in Section 3 are shown in Figure 8. The results are normalized relative to the LOS signal which is the first position where the Tx and Rx antennas have a LOS path when walking starts. We can clearly observe the periodic shadowing effect of the body during one walking cycle. We can also observe that the shadowing effects of the right-belt-to-left-wrist and right-belt-to-left-ankle are opposite to each other (during walking left wrist sways in opposite direction to left ankle), as expected. These fading behaviors are similar to the ones observed in reported measurement results such those in $[1,3,10]$. Furthermore, it is known from measurements that the shadowing (in $\mathrm{dB}$ ) of the signal by human body part follows a normal

Table 5 Simulation parameters

\begin{tabular}{lc}
\hline Parameter & Value \\
\hline Frequency, $f$ & $2.4 \mathrm{GHz}$ \\
Sampling frequency, $f_{\mathrm{s}}$ & $10 \mathrm{~Hz}$ \\
Rice K-factor, $K$ & $7 \mathrm{~dB}$ \\
TX and Rx dipole antenna length, $L$ & 0.5 and $1.5 \lambda$ \\
Human body dimension & See Table 4 \\
Relative waking velocity, $V_{\mathrm{r}}$ & 1 \\
Number of walking cycles, $N$ & 1 and 100 \\
\hline
\end{tabular}

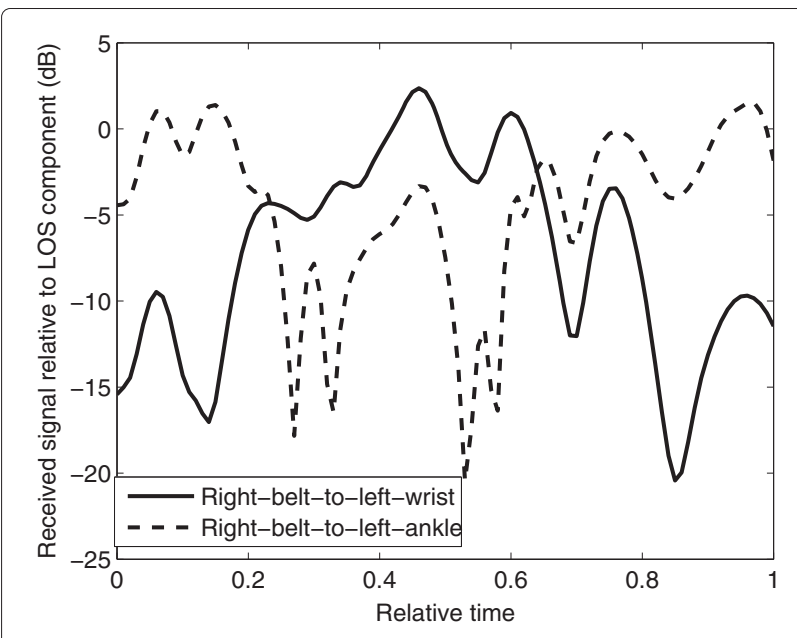

Figure 8 Simulated time series of the received signal at $2.4 \mathrm{GHz}$ for the right-belt-to-left-wrist and right-belt-to-left-ankle links. This is for one walking cycle. The human body dimension shown in Table 4 and the simulation parameters given in Table 5 are used.

distribution [1-3,7]. Figure 9 shows comparisons between the cumulative distribution functions (CDFs) of the normal function and the simulated right-belt-to-left-wrist signal. We can observe from Figure 9 that there is relatively good agreement between the CDFs. In addition, Figure 10a,b shows the simulated level crossing rates (LCRs) and average fade durations (AFD) of the received signal at $2.4 \mathrm{GHz}$ for the right-belt-to-left-wrist and rightbelt-to-left-ankle links. We can observe that the LCR of the right-belt-to-left-ankle link is higher than that of the right-belt-to-left-wrist link. This might be due the fact that the displacement of the ankle during walking is larger

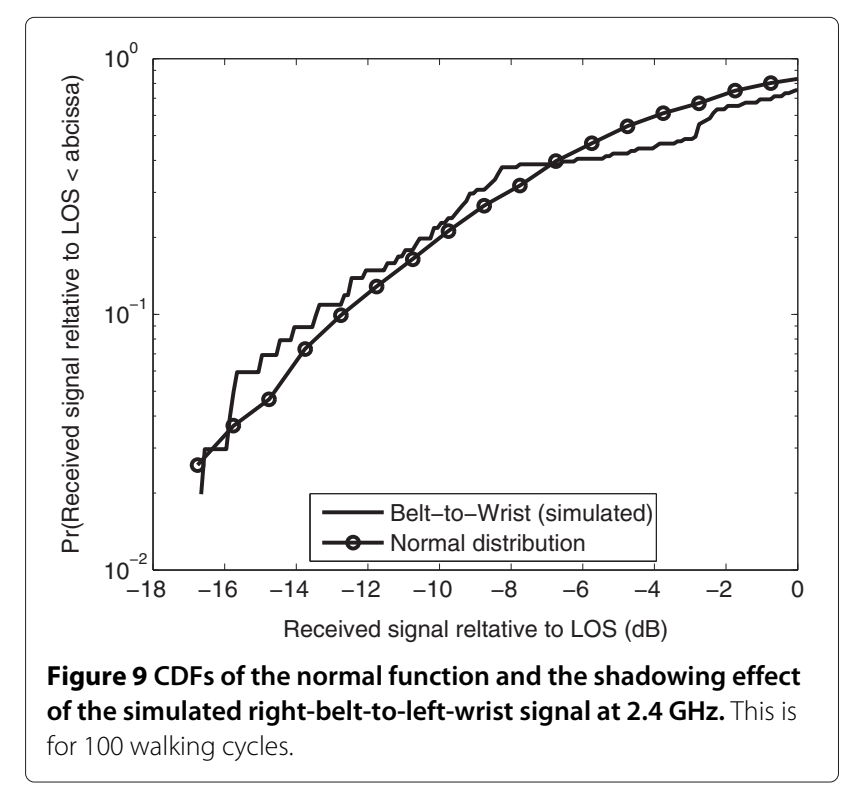




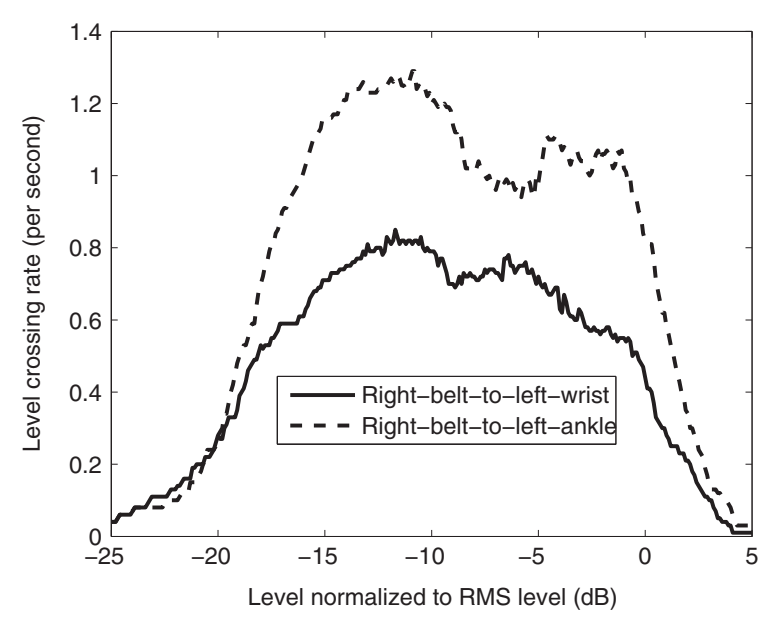

(a)

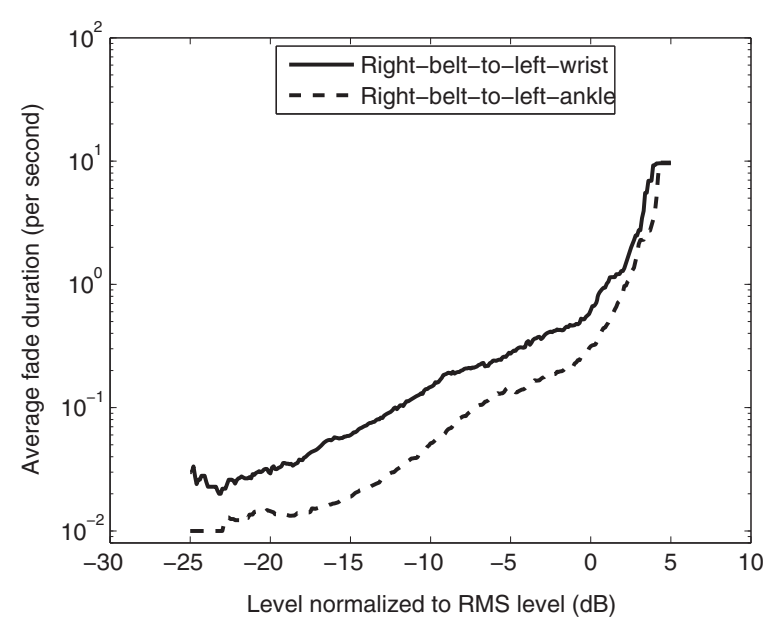

(b)

Figure 10 Simulated LCRs (a) and AFDs (b) of the received signal at $2.4 \mathrm{GHz}$ for 100 walking cycles. This is for the right-belt-to-left-wrist and right-belt-to-left-ankle links. The human body dimension shown in Table 4 and the simulation parameters given in Table 5 are used.

than that of the wrist, resulting in large signal variations, thereby higher LCR. On the other hand, the fade duration of the right-belt-to-left-wrist is larger than that of the right-belt-to-left-ankle link (see Figure 10b). Again, this might be due to the shorter displacement of the wrist compared to the ankle (during walking), resulting in being in NLOS positions for a longer period of time.

As discussed in Section 3.4, because of body movements, a wearable antenna is constantly changing its direction of maximum radiation, which leads to timevarying channel conditions. To demonstrate this, two dipole antennas with different lengths $(L=0.5 \lambda$ and $L=1.5 \lambda$, where $\lambda$ is the wavelength) having different radiation patterns are considered. The antennas are matched and are vertically mounted on the right belt and on the left wrist of the body. The antenna gain variations are calculated using (15), where the angular rotations of the corresponding body parts are used. Figure 11 shows the received signal CDFs, LCRs, and AFDs when both the transmitter and receiver antennas are 0.5 and $1.5 \lambda$ dipole antennas for the right-belt-to-left-wrist link, respectively. We can observe from Figure 11 that the effect of antenna gain variations strongly depend on the radiation pattern of the antennas and has a significant effect on the first- and second-order statistics of the signal (note that the effect of the body on the radiation pattern of the antenna is not considered).

\subsection{Performance evaluation}

As shown in Figure 4 the different body parts have different flexing angles during walking. These differences in flexing angles might be exploited to increase the system performance using diversity techniques. For example, for the time series shown in Figure 8, the right-belt-to-leftankle link is on the LOS position (not shadowed) when the right-belt-to-left-wrist link is shadowed, and vice versa. If we now assume that the receiver is located on the belt toward the right side of the body and two transmitting positions (on the wrist and ankle), the time series may now be combined using, e.g., selection combing to improve the link availability. For diversity with selection combing, the link with maximum signal strength $S_{i}($ for $i=1,2)$ is selected at any time, that is, $S_{\mathrm{d}}=\max \left(S_{1}, S_{2}\right)$. Figure 12 presents the CDFs of the received signal for the right-beltto-left-ankle link $\left(S_{1}\right)$, right-belt-to-left-wrist link $\left(S_{2}\right)$, and the diversity link $\left(S_{\mathrm{d}}\right)$. We can observe the significant gain achieved using diversity compared to single link cases. In general, as the flexing of the different body parts exhibits different angular variations, choosing properly diversity antenna locations may increase the system performance (i.e., during walking, at any given time, one diversity link might be on LOS condition while the other links are shadowed by body parts).

\section{Conclusion}

The on-body wireless channel is subject to fading caused by the movement of the human body parts and by the reflection/scattering of the signal by objects in the vicinity of the human body. These effects need to be taken into account for a realistic characterization of the channel. In this contribution, a novel dynamic channel model for on-body wireless communication during walking is proposed. The developed model uses a human walking model which provides detailed information on the movement of the human body parts. Using geometrical relations, the diffraction of the signal around the body parts is calculated to describe the time-varying shadowing effects. The CDFs of these shadowing effects follow a normal 


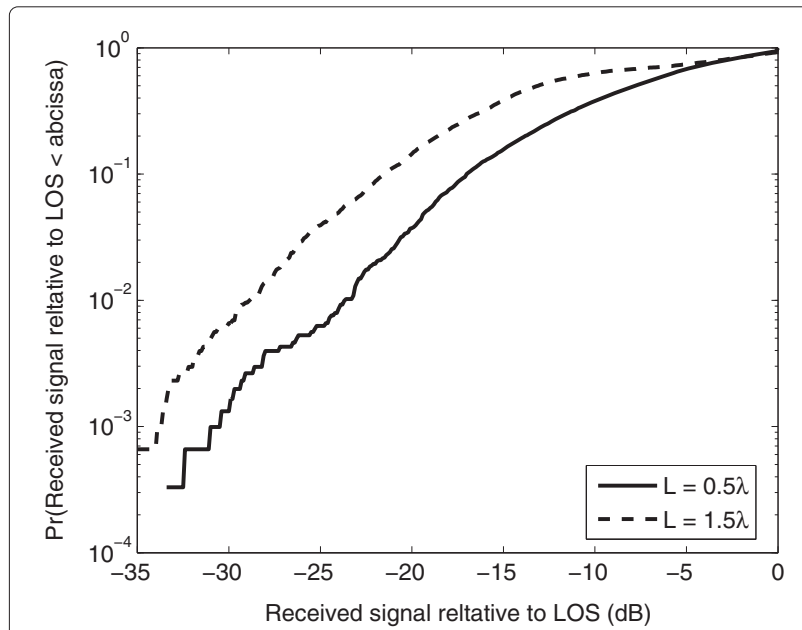

(a)

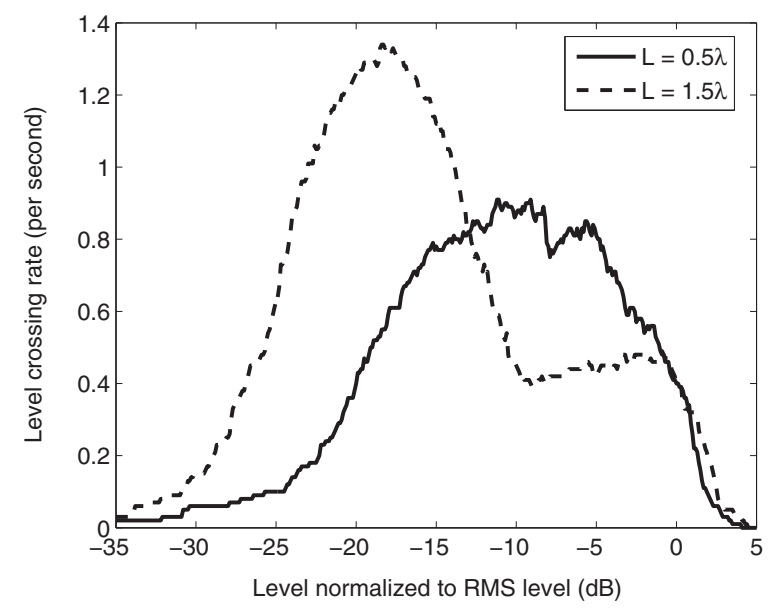

(b)

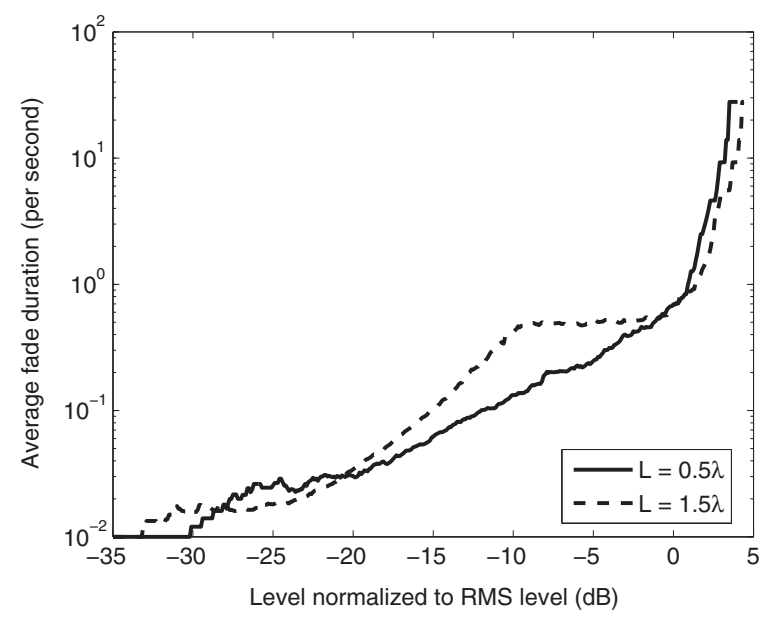

(c)

Figure 11 Simulated CDFs (a), LCRs (b), and AFDs (c) of the received signal at $2.4 \mathrm{GHz}$. These are for 100 walking cycles for the right-belt-to-left-wrist for different dipole antenna lengths $(L=0.5 \lambda$ and $L=1.5 \lambda$ ). The human body dimension shown in Table 4 and the simulation parameters given in Table 5 are used.

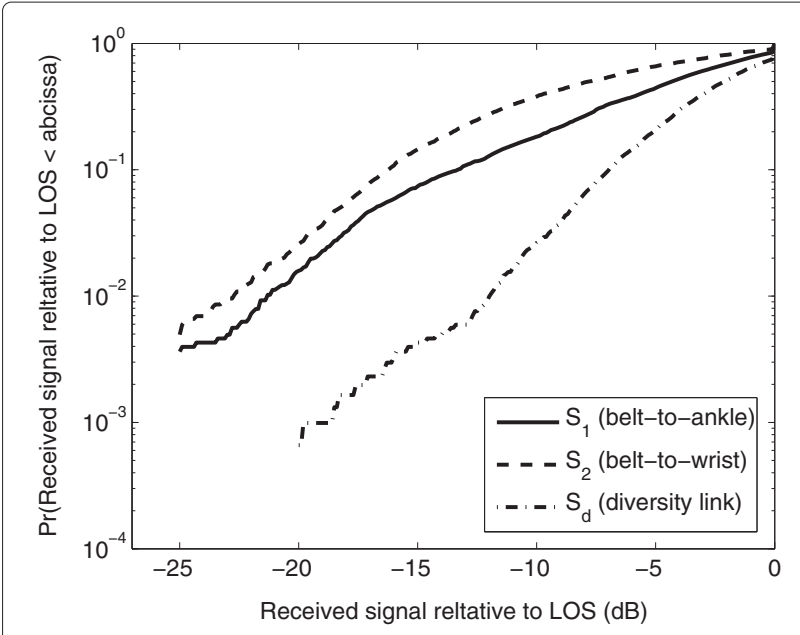

Figure $12 \mathrm{CDFs}$ of the received signal at $2.4 \mathrm{GHz}$ for 100 walking cycles. These are for the right-belt-to-left-ankle $\left(S_{1}\right)$, right-belt-to-leftwrist $\left(S_{2}\right)$, and diversity $\left(S_{d}\right)$ links. The human body dimension shown in Table 4 and the simulation parameters given in Table 5 are used.

distribution which is consistent with the reported measurement results. A Rice distribution is used to represent the fast fading effects caused by objects around the human body, where the Rice K-factor depends on the mobility of the human body. High value of Rice K-factor is expected when the human subject is at rest, while low K-factor is expected in a walking scenario. The model takes also into account the Doppler effects caused by the movement of the different body parts.

Body part movements are also used to estimate the signal fading caused by angular variations of the antenna gains. It is found that the effect of antenna gain variations strongly depends on the radiation pattern of the antennas and has a significant effect on the first- and second-order statistics of the signal.

Furthermore, the simulation results of the first- and second-order statistics of the received signal at $2.4 \mathrm{GHz}$ for the right-belt-to-left-wrist and right-belt-to-left-ankle links are presented. In addition, to illustrate the capabilities of the developed model, time series were generated and used in system performance calculations. The obtained results suggest that choosing properly diversity antenna locations may increase the system performance, giving an insight into the potential advantages of link diversity technique in WBANs.

In general, the proposed dynamic channel model can be used to carry out different system-level analyses such as capacity and bit error rate for on-body wireless communication. Future work includes validating the developed time-varying on-body wireless channel model during walking using measurements. In addition, the model will be extended covering the body effect on antenna and its 
polarization, a better off-body multipath description and the random properties of human kinetics.

\section{Competing interests}

The author declares that he has no competing interests.

\section{Acknowledgements}

The author would like to thank Gjøvik University College, Norway, for supporting this work.

\section{Received: 6 May 2013 Accepted: 6 February 2014}

Published: 20 February 2014

\section{References}

1. R DErrico, L Ouvry, A statistical model for on-body dynamic channels. Int. J. Wireless Inf. Netw. 17, 92-104 (2010)

2. YI Nechayev, PS Hall, I Khan, CC Constantinou, Wireless channels and antennas for body-area networks. Paper presented at the 7th international conference on wireless on-demand network systems and services (WONS) (Kranjska Gora, 3-5 Feb 2010), pp. 137-144

3. IEEE P802.15 Working Group for Wireless Personal Area Networks, IEEE P802.15: Statistical Property of Dynamic BAN Channel Gain at 4.5GHz. (IEEE, Piscataway, 2008)

4. E Reusens, W Joseph, B Latre, B Braem, G Vermeeren, E Tanghe, L Martens, I Moerman, C Blondia, Characterization of on-body communication channel and energy efficient topology design for wireless body area networks. IEEE Tran. Inf. Tech. Bio. 13(6), 933-945 (2009)

5. B Zhen, M Kim, J Takada, R Kohno, Finite-state Markov model for on-body channels with human movements. Paper presented at the, IEEE international conference on communications (ICC) (Cape Town, 23-27 May 2010)

6. A Taparugssanagorn, B Zhen, R Tesi, M Mamalainen, J linatti, R Kohno, A dynamic channel model of UWB-WBAN for some medical applications. Paper presented at the wireless personal multimedia communications symposium (Lapland, 8-11 Sept 2008)

7. L Liu, R Derrico, L Ouvry, PD Doncker, C Oestges, Dynamic channel modelling at $2.4 \mathrm{GHz}$ for on-body area networks. Adv. Wireless Commun. Netw. 2(4) (2011)

8. SV Roy, F Quitin, L Liu, C Oestges, F Horlin, J Dricot, PD Doncker, Dynamic channel modelling for multi-sensor body area networks. IEEE Tran. Ant. Prop. 61(4), 2200-2208 (2013)

9. C Roblin, J Laheurte, Derrico R, A Gati, D Lautru, T Alves, H Terchoune, F Bouttout, Antenna design and channel modelling in the BAN context-part II: channel. Ann. Telecommun. 66, 157-175 (2011)

10. T Aoyagi, MK Iswandi, J Takada, K Hamaguchi, R Kohno, Body motion and channel response of dynamic body area channel. Paper presented at the 5th European conference on antennas and propagation (EUCAP) (Rome, 11-15 Apr 2011)

11. Iswandi, M Kim, J Takada, Analysis of antenna motion dependency on BAN channels. IEICE Technical Report (MICT2010) (Tokyo, 2010)

12. WG Scanlon, GA Conway, SL Cotton, Antennas and propagation considerations for robust wireless communications in medical body area networks. Paper presented at IET seminar on antennas and propagation body-centric wireless communications (London, 24 Apr 2007)

13. S Swaisaenyakron, P Young, J Batchelor, Animated human walking movement for body worn antenna study. Paper presented at the Loughborough antennas and propagation conference (Loughborough, 14-15 Nov 2011)

14. Q Wang, T Tayamichi, I Kimura, J Wang, An on-body channel model for UWB body area communications for various postures. IEEE Tran. Ant. Prop. 4(57), 991-998 (2009)

15. M Gallo, PS Hall, YI Nechayev, M Bozzetti, Use of animation software in simulation of on-body communications channels at $2.45 \mathrm{GHz}$. IEEE Ant. Wireless Prop. Lett. 7, 321-324 (2008)

16. K Ogawa, K Honda, BAN shadowing properties of an arm-waving dynamic phantom. Paper presented at the 5th European conference on antennas and propagation (EUCAP) (Rome, 11-15 Apr 2011)

17. F Thomas, O Johanston, The illusion of Life: Disney Animation, (New York, 1995)

18. TWhite, The Animator's Workbook. (Watson-Guptill, New York, 1988)
19. R Boulic, N Magnenat-Thalmann, D Thalmann, A global human walking model with real-time kinematic personification. Vis. Comput. 6, 344-358 (1990)

20. P Dorp, FCA Groen, Human walking estimation with radar. IEE Proc. Radar Sonar Navig. 150(5), 356-365 (2003)

21. HD Hristov, Fresnel Zones in Wireless Links, Zone Plate Lenses and Antennas. (Artech House, Norwood, 2000)

22. FP Fontan, A Abele, B Montenegro, F Lacoste, V Fabbro, L Castanet, B Sanmartin, P Valtr, Modelling of the land mobile satellite channel using a virtual city approach. Paper presented at the 2nd European conference on antennas and propagation (EUCAP) (Edinburgh, 11-16 Nov 2007)

23. F Perez-Fontan, PM Espineira, Modelling the Wireless Propagation Channel: A Simulation Approach with MATLAB. (Wiley, Chichester, 2008)

24. RH Clarke, A statistical theory of mobile radio reception. Bell Syst. Tech. J. 47, 957-1000 (1968)

25. RJC Bultitude, Measurement characterization and modelling of indoor 800/900 MHz radio channels for digital communications. IEEE Commun. Meg. 25(6), 5-12 (1987)

26. SJ Howard, K Pahlavan, Doppler spread measurements of indoor radio channel. Electron. Lett. 26(2), 107-109 (1990)

27. JB Andersen, JO Nielsen, GF Pedersen, G Bauch, Doppler spectrum from moving scatterers in a random environment. IEEE Trans. Wirl. Commun. 8(6), 3270-3276 (2009)

28. M Mackowiak, LM Correia, A statistical model for the influence of body dynamics on the gain pattern of wearable antennas in off-body radio channels. Wireless Pers. Commun. 73, 381-399 (2013). doi:10.1007/s11277-013-1193-x

doi:10.1186/1687-1499-2014-29

Cite this article as: Cheffena: Time-varying on-body wireless channel model during walking. EURASIP Journal on Wireless Communications and Networking 2014 2014:29.

\section{Submit your manuscript to a SpringerOpen ${ }^{\circ}$ journal and benefit from:}

- Convenient online submission

Rigorous peer review

- Immediate publication on acceptance

- Open access: articles freely available online

- High visibility within the field

- Retaining the copyright to your article

Submit your next manuscript at $>$ springeropen.com 